\title{
ETHzürich
}

\section{On the use of one-step}

perturbation to investigate the dependence of NOE-derived atomatom distance bound violations of peptides upon a variation of forcefield parameters

\section{Journal Article}

\section{Author(s):}

Lin, Zhixiong; Oostenbrink, Chris; van Gunsteren, Wilfred F.

Publication date:

2014-03

Permanent link:

https://doi.org/10.3929/ethz-b-000083886

Rights / license:

In Copyright - Non-Commercial Use Permitted

Originally published in:

European Biophysics Journal 43(2-3), https://doi.org/10.1007/s00249-014-0943-3

Funding acknowledgement:

137827 - Biomolecular Simulation of Biomolecular Systems II (SNF) 


\title{
On the use of one-step perturbation to investigate the dependence of NOE-derived atom-atom distance bound violations of peptides upon a variation of force-field parameters
}

\author{
Zhixiong Lin • Chris Oostenbrink • \\ Wilfred F. van Gunsteren
}

Received: 23 October 2013 / Revised: 13 January 2014 / Accepted: 17 January 2014 / Published online: 7 February 2014

(C) European Biophysical Societies' Association 2014

\begin{abstract}
The method of one-step perturbation can be used to predict from a single molecular dynamics simulation the values of observable quantities as functions of variations in the parameters of the Hamiltonian or biomolecular force field used in the simulation. The method is used to predict violations of nuclear overhauser effect (NOE) distance bounds measured in nuclear magnetic resonance (NMR) experiments by atom-atom distances of the NOE atom pairs when varying force-field parameters. Predictions of NOE distance bound violations between different versions of the GROMOS force field for a hexa$\beta$-peptide in solution show that the technique works for rather large force-field parameter changes as well as for very different NOE bound violation patterns. The effect of changing individual force-field parameters on the NOE distance bound violations of the $\beta$-peptide and an $\alpha$-peptide was investigated too. One-step perturbation, which in this case is equivalent to reweighting configurations, constitutes an efficient technique to predict many values of different quantities from a single conformational ensemble for a particular system, which makes it a powerful force-field development technique that easily reduces the number of required separate simulations by an order of magnitude.
\end{abstract}

\footnotetext{
Z. Lin · W. F. van Gunsteren $(\square)$

Laboratory of Physical Chemistry, Swiss Federal Institute

of Technology, ETH, 8093 Zurich, Switzerland

e-mail:wfvgn@igc.phys.chem.ethz.ch

C. Oostenbrink

Institute for Molecular Modeling and Simulation, University

of Natural Resources and Life Sciences (BOKU), Vienna, Austria
}

Keywords Molecular dynamics (MD) simulation . One-step perturbation $\cdot$ Reweighting $\cdot$ NOE distance bound violations $\cdot$ Peptide

Molecular dynamics (MD) simulation represents a useful tool for investigating the properties of molecular systems relevant in physics, chemistry, and biology. However, the quality of the results obtained from MD simulations depends on the extent of sampling of the conformational space accessible to a molecular system and on the accuracy of the underlying force field (van Gunsteren and Berendsen 1990). A major effort in the realm of molecular simulation is thus directed towards the parametrization and validation of force fields against available experimental data (Cornell et al. 1995; Jorgensen et al. 1996; MacKerell et al. 1998; Reif et al. 2012).

The parametrization philosophy of the GROMOS force field (van Gunsteren et al. 2006; van Gunsteren et al. http://www.gromos.net) is to determine as many values of the parameters as possible by fitting particular properties of small molecules in the condensed phase as simulated to corresponding experimental values (Horta et al. 2011), and to subsequently validate the force field against experimental data of biomolecular systems such as peptides, proteins, nucleic acids, carbohydrates, and lipids. To avoid jeopardizing the transferability of parameters between groups of atoms in different molecules, data on large molecules are not used in the parametrization phase. If one were to use such data from molecules such as proteins, e.g., structures from the Protein Data Bank, properties of groups of atoms may well be dependent on their particular environment in the folded molecule. Furthermore, the Protein Data Bank contains structures derived from measurements at different thermodynamic conditions of $\mathrm{pH}$, ionic strength, etc. Using 
the GROMOS force field, generally, good agreement with experimental data of biomolecules was obtained; see, for example, a recent validation of the new GROMOS 54A8 force field (Reif et al. 2013). However, in case experimental data for test molecules are not reproduced, e.g., the conformational preference of a hexa- $\beta$-peptide in solution was shown to be wrongly described by the GROMOS 54A7 force field (Lin and van Gunsteren 2013), the responsible force-field parameter is to be identified. This could be done by running additional simulations with different values of force-field parameters and different combinations thereof, which is, however, computationally very costly. In this communication, we illustrate a technique that can give an indication of which force-field parameters should be changed without the need of repeating simulations.

One-step perturbation (Liu et al. 1996) is an efficient method to calculate many free energy differences from a single simulation (Oostenbrink 2012). It can, however, also be applied to predict structural properties from a single simulation (Oostenbrink and van Gunsteren 2005; Hritz and Oostenbrink 2009; Li and Brüschweiler 2010; Bachmann et al. 2013). Examples are the occurrence of hydrogen bonds (Oostenbrink and van Gunsteren 2005), NMR ${ }^{3}$ J-coupling constants (Hritz and Oostenbrink 2009), and properties of liquid water (Bachmann et al. 2013). The same idea was also applied to optimize the Amber ff99SB force field with respect to chemical shifts of proteins $(\mathrm{Li}$ and Brüschweiler 2010).

Using one-step perturbation, structural properties can be predicted as follows. For a quantity $Q\left(\vec{r}^{N}\right)$ that depends on the coordinates $\vec{r}^{N}=\left(\vec{r}_{1}, \vec{r}_{2}, \ldots, \vec{r}_{N}\right)$ of a system of $N$ particles, the average $\langle Q\rangle_{\mathrm{A}}$ of the quantity $Q\left(\vec{r}^{N}\right)$ calculated over the ensemble of configurations $\vec{r}^{N}$ generated using the Hamiltonian $H_{\mathrm{A}}$ can be predicted from a reference simulation of the reference Hamiltonian $H_{\mathrm{R}}$,

$\langle Q\rangle_{\mathrm{A}}=\frac{\left\langle Q \mathrm{e}^{-\left(H_{\mathrm{A}}-H_{\mathrm{R}}\right) / k_{\mathrm{B}} T}\right\rangle_{\mathrm{R}}}{\left\langle\mathrm{e}^{-\left(H_{\mathrm{A}}-H_{\mathrm{R}}\right) / k_{\mathrm{B}} T}\right\rangle_{\mathrm{R}}}$,

in which $\langle\cdots\rangle_{R}$ denotes the ensemble average over the configurations sampled in the reference simulation. In this way, the quantity $\langle Q\rangle_{\mathrm{A}}$ for many different Hamiltonians $H_{\mathrm{A}}$ can be predicted from a single simulation using the reference Hamiltonian $H_{\mathrm{R}}$. This application of one-step perturbation consists of reweighting the configurations $\vec{r}^{N}$ of the ensemble $\mathrm{R}$ with the factor $\mathrm{e}^{-\left(H_{\mathrm{A}}\left(\vec{r}^{N}\right)-H_{\mathrm{R}}\left(\vec{r}^{N}\right)\right) / k_{\mathrm{B}} T} / \sum \mathrm{e}^{-\left(H_{\mathrm{A}}\left(\vec{r}^{N}\right)-H_{\mathrm{R}}\left(\vec{r}^{N}\right)\right) / k_{\mathrm{B}} T}$ and is similar to the unbiasing step in umbrella sampling (Torrie and Valleau 1977).

The accuracy of Eq. (1) in predicting structural properties depends on the overlap of the configurations belonging to $H_{\mathrm{A}}$ and $H_{\mathrm{R}}$, and on the accuracy of the function $Q\left(\vec{r}^{N}\right)$ that connects the observable $Q$ to a configuration $\vec{r}^{N}$.
Regarding the latter, the relation between a chemical shift and a molecular structure is not completely understood, and the Karplus relation ${ }^{3} \mathbf{J}(\theta)$ relating a ${ }^{3} \mathbf{J}$-coupling constant to a torsional angle $\theta$ has a large statistical uncertainty (Steiner et al. 2012). On the other hand, a NOE intensity is related to the atom-atom distance $r_{i j}$ of the atoms $i$ and $j$ involved in the NOE,

$Q\left(r_{i j}\right) \propto r_{i j}^{-6}$.

So, this quantity $Q$ will be used in this study. For a protein, the set of NOE atom-atom distances may not be very sensitive to changes in force-field parameters as long as the particular fold of the protein is maintained (Eichenberger et al. 2010). For small flexible peptides though, different conformations may be present in the ensemble and may contribute to $\langle Q\rangle_{\mathrm{A}}$, and the relative occurrence of the different conformations is generally sensitive to force-field parameter changes.

Based on the above considerations, we use one-step perturbation or reweighting to predict the NOE distance bound violations for a hexa- $\beta$-peptide (Seebach et al. 1998; Daura et al. 1999) $\left(\mathrm{H}_{2}^{+}-\beta^{2}-\right.$ HVal $-\beta^{3}$-HAla- $\beta^{2}$-HLeu- $\beta^{3}-$ HVal- $\beta^{2}-$ HAla- $\beta^{3}-\mathrm{HLeu}-\mathrm{OH}$ ) and a hepta- $\alpha$-peptide (Seebach et al. 2005; Wang et al. 2010) (Val-Ala-Leu-Aib-Ile-Met-Phe). The accuracy of the technique is first tested through perturbations between different GROMOS force fields for the $\beta$-peptide. Next, the method is used to predict the effect of changing individual force-field parameters on the NOE distance bound violations of both peptides.

The simulations of the two peptides were carried out in explicit methanol solvent using the GROMOS11 simulation package (van Gunsteren et al. http://www.gromos.net; Eichenberger et al. 2011; Riniker et al. 2011; Schmid et al. 2011a, 2012). The simulation temperatures and lengths are listed in Table 1. The details of the simulation setup are described by Lin and van Gunsteren (2013) for the $\beta$-peptide and Wang et al. (2010) for the $\alpha$-peptide. Interproton distance bounds derived from the NOE cross-peak

Table 1 Overview of the MD simulations

\begin{tabular}{llllll}
\hline Peptide & $\begin{array}{l}\text { Simulation } \\
\text { name }\end{array}$ & $\begin{array}{l}\text { Force } \\
\text { field }^{\mathrm{a}}\end{array}$ & $\begin{array}{l}\text { No. solvent } \\
\text { molecules }\end{array}$ & $T(\mathrm{~K})$ & $\begin{array}{l}\text { Simulation } \\
\text { time (ns) }\end{array}$ \\
\hline $\mathrm{P} \beta$ & $P \beta \_45 A 3$ & $45 \mathrm{~A} 3$ & 1,123 & 340 & 500 \\
& $P \beta \_53 A 6^{* \mathrm{~b}}$ & - & 1,123 & 340 & 500 \\
& $P \beta \_53 A 6$ & $53 \mathrm{~A} 6$ & 1,123 & 340 & 500 \\
& $P \beta \_54 A 7$ & $54 \mathrm{~A} 7$ & 1,123 & 340 & 500 \\
$\mathrm{P} \alpha$ & $P \alpha \_53 A 6$ & $53 \mathrm{~A} 6$ & 1,556 & 300 & 200 \\
\hline
\end{tabular}

a GROMOS force fields used for the peptides: $45 \mathrm{~A} 3$ (Schuler et al. 2001), 53A6 (Oostenbrink et al. 2004), 54A7 (Schmid et al. 2011b)

b The 53A6 force field was used for the $\beta$-peptide, and $45 \mathrm{~A} 3$ for the methanol solvent 
intensities (Seebach et al. 1998, 2005) were compared with the corresponding average effective interproton distances in the simulations, calculated as $\left\langle r^{-6}\right\rangle^{-1 / 6}$. The hydrogenhydrogen distances involving aliphatic hydrogen atoms were calculated by defining virtual $\left(\mathrm{CH}_{1}\right)$, prochiral (stereospecific $\left.\mathrm{CH}_{2}\right)$, and pseudo $\left(\mathrm{CH}_{3}\right.$ and nonstereospecific $\mathrm{CH}_{2}$ ) atomic positions, and the distance bounds for the latter were modified to include pseudo-atom distance bound corrections $\left(0.10 \mathrm{~nm}\right.$ for nonstereospecific $\mathrm{CH}_{2}, 0.15 \mathrm{~nm}$ for $\mathrm{CH}_{3}$, and $0.29 \mathrm{~nm}$ for nonstereospecific rotating methyls) (Wüthrich et al. 1983).

The NOE distance bound violations out of the $32 \mathrm{NOE}$ distance bounds (Seebach et al. 1998) of the $\beta$-peptide using different GROMOS force fields are shown in Fig. 1a, $\mathrm{d}$, and $\mathrm{g}$ for GROMOS 45A3, 53A6, and 54A7, respectively. The 45A3 (Schuler et al. 2001) and 53A6 (Oostenbrink et al. 2004) force fields have sizable differences in their parameter values of the backbone atom partial charges (up to $20 \%$ ), terminal group partial charges (up to $50 \%$ ), as well as the van der Waals parameters of the polar backbone atoms (up to $20 \%$ ), yet the simulations

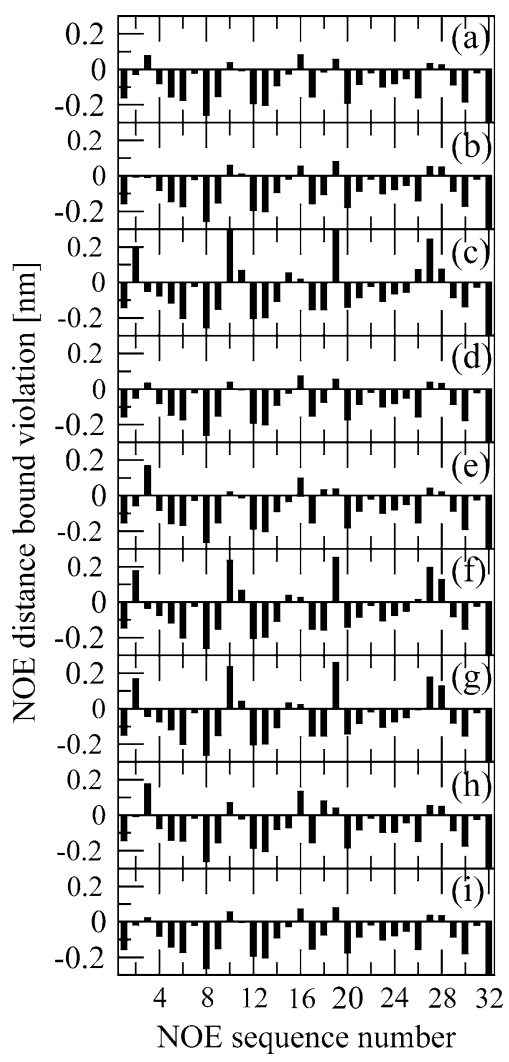

Fig. 1 Comparison of $r^{-6}$ averaged NOE distances as obtained from simulations or predicted by one-step perturbation and experimental data for $P \beta$. a $P \beta \_45 A 3$; b 53A6 predicted by $P \beta \_45 A 3$; c 54A7 predicted by $P \beta \_45 A 3$; d $P \beta \_53 A 6$; e $45 \mathrm{~A} 3$ predicted by $P \beta \_53 A 6$; f 54A7 predicted by $P \beta \_53 A 6 ; \mathbf{g} P \beta \_54 A 7 ; \mathbf{h} 45 \mathrm{~A} 3$ predicted by $P \beta \_54 A 7 ;$ i 53 A6 6 predicted by $P \beta \_54 A 7$ of the $\beta$-peptide using these two force fields show a very similar picture of NOE bound violations; i.e., for both force fields, there are six positive violations $<0.1 \mathrm{~nm}$ for the same hydrogen-hydrogen distances (Lin and van Gunsteren 2013). In contrast, the 54A7 force field (Schmid et al. 2011b) gives a completely different violation pattern with five positive NOE bound violations larger than $0.1 \mathrm{~nm}$ (Lin and van Gunsteren 2013), although it only differs from 53A6 (Oostenbrink et al. 2004) in the backbone $\varphi$ and $\psi$ torsional angle energy terms and the backbone $\mathrm{N}$ and $\mathrm{O}$ van der Waals interactions for this $\beta$-peptide.

Figure 2 shows a comparison of NOE bound violations of the $\beta$-peptide predicted by one-step perturbation and evaluated from direct MD simulations. Since 45A3 has a different solvent methanol model, an additional simulation was carried out with 53A6 as the solute force field, and $45 \mathrm{~A} 3$ as the methanol solvent force field (Table 1). This allows for determination of the effect of changing only the solute force-field parameters. According to Fig. 2, the prediction by one-step perturbation is very accurate; i.e., the deviations between the predicted violations and the simulated ones are mostly within $0.02 \mathrm{~nm}$. The accurate prediction when perturbing from $45 \mathrm{~A} 3$ to $53 \mathrm{~A} 6$ shows that the technique works for rather large force-field parameter changes, and the perturbation between 53A6 and 54A7 illustrates that the technique works for very different $\mathrm{NOE}$ bound violation patterns. We note in passing that the 53A6 force field (blue and green symbols in Fig. 2) yields much

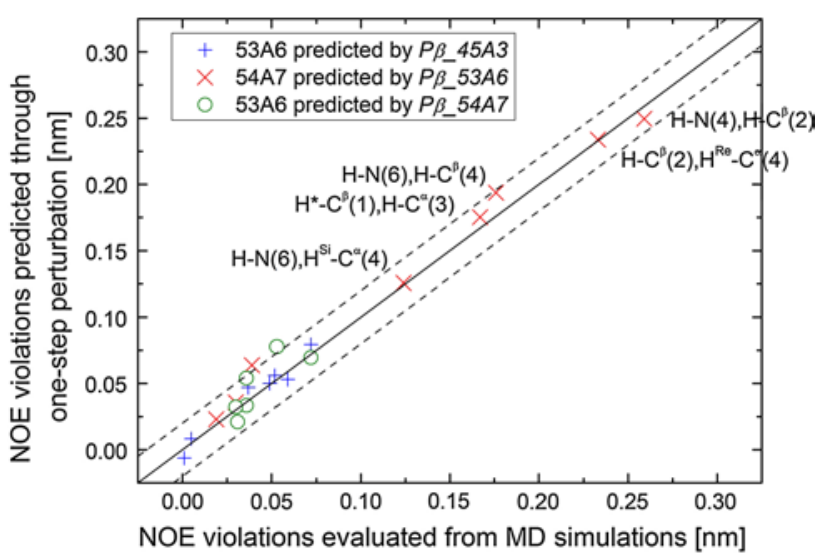

Fig. 2 Comparison of NOE atom-atom distance bound violations of the $\beta$-peptide predicted by one-step perturbation and evaluated from MD simulation. Blue plus sign NOE violations predicted by $P \beta \_45 A 3$ compared with the ones evaluated from $P \beta \_53 A 6^{*}$; red cross NOE violations predicted by $P \beta \_53 A 6$ compared with the ones evaluated from $P \beta \_54 A 7$; green circle NOE violations predicted by $P \beta \_54 A 7$ compared with the ones evaluated from $P \beta \_53 A 6$. The dashed lines indicate a deviation of $0.02 \mathrm{~nm}$. The hydrogen atom pairs with violations larger than $0.1 \mathrm{~nm}$ are listed. $\mathrm{H}^{*}-\mathrm{C}^{\beta}$ refers to nonstereospecific protons bound to $\mathrm{C}^{\beta}$ 
Fig. 3 Sum of the positive NOE distance bound violations for the $\beta$-peptide predicted by one-step perturbation using the $P \beta \_53 A 6$ simulation as the reference state and varying different force-field parameters
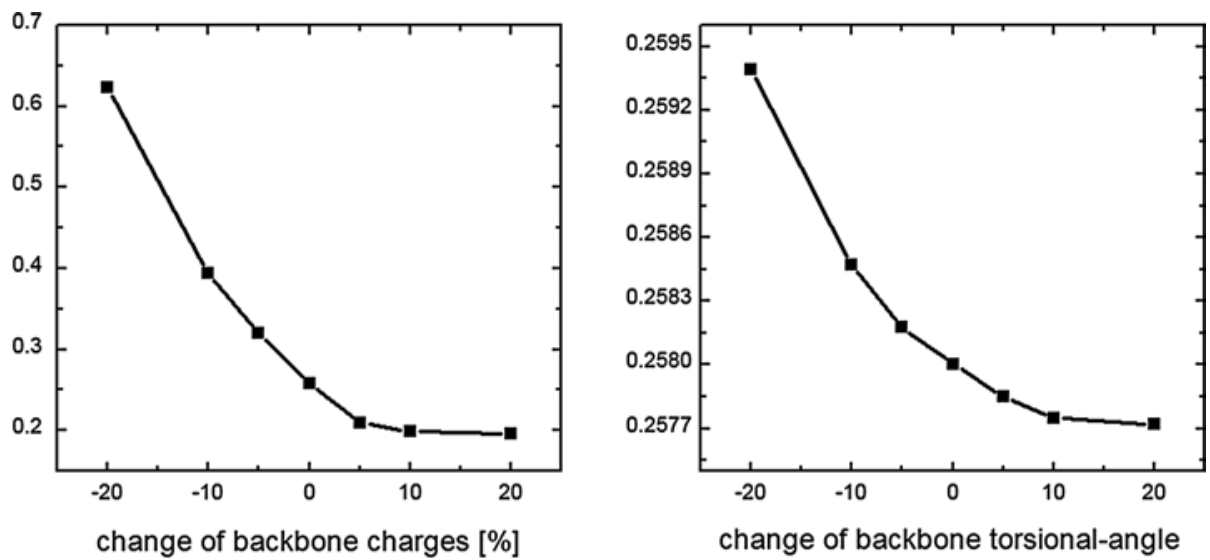

change of backbone torsional-angle force constants [\%]
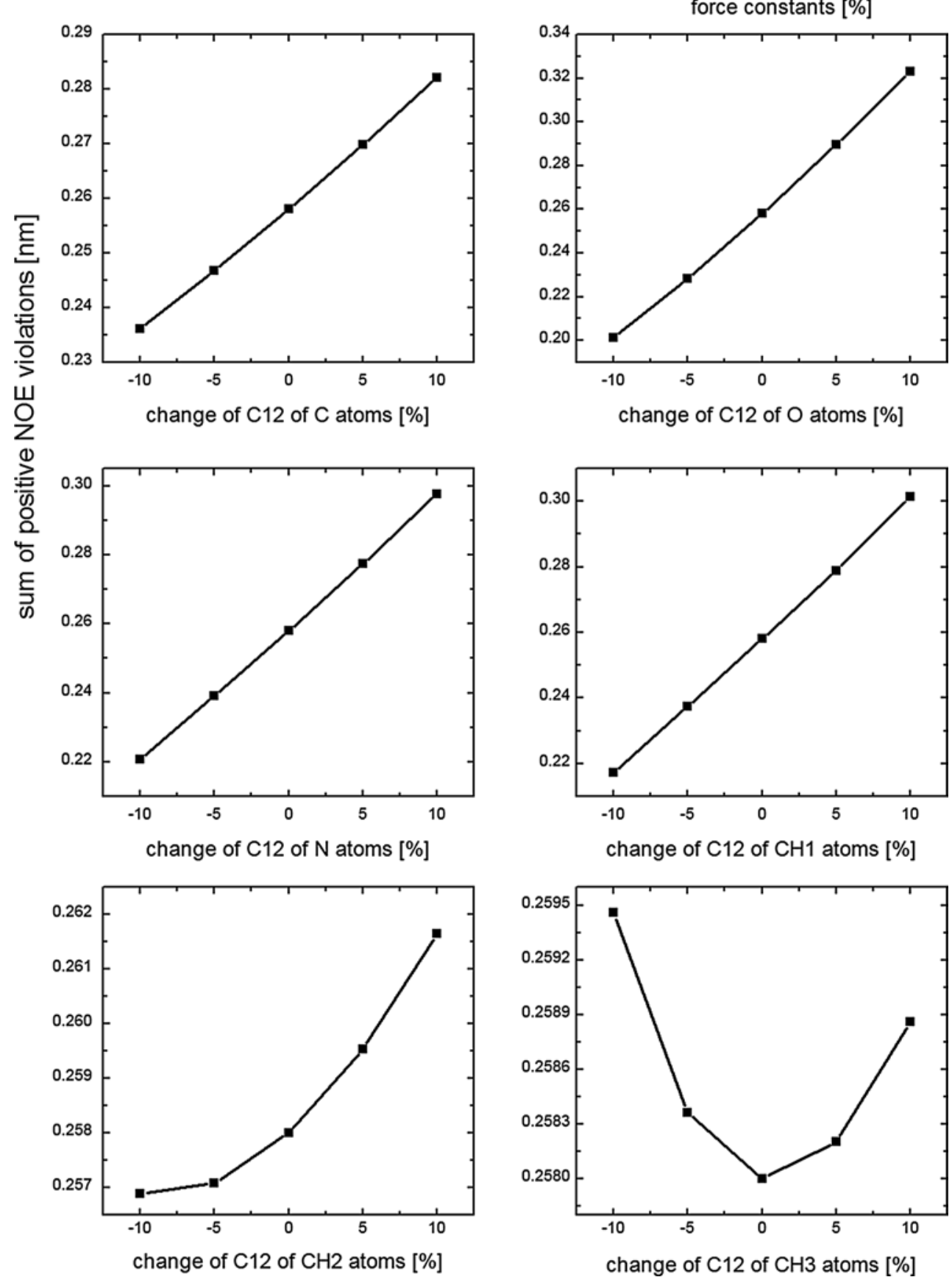

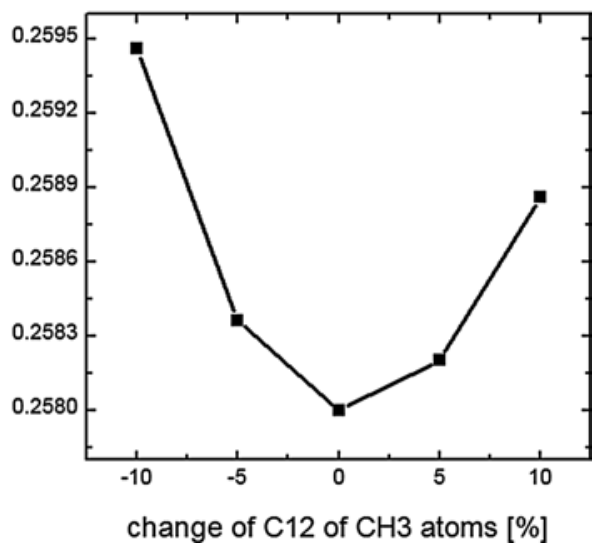


smaller violations than the 54A7 one (red symbols in Fig. 2).

In a previous study, Bachmann et al. (2013) concluded that one-step perturbation can only be used to qualitatively predict different properties of liquid water. In contrast, the NOE distance bound violations of the $\beta$-peptide can be predicted using one-step perturbation not only qualitatively, but also quantitatively. The less accurate prediction in the previous study is related to the larger size of the perturbation considered in that study, i.e., changing force-field parameters by $5 \%$ for up to 5,000 water molecules (Bachmann et al. 2013).

Fig. 4 Sum of the positive NOE distance bound violations for the $\alpha$-peptide predicted by one-step perturbation using the $P \alpha \_53 A 6$ simulation as the reference state and varying different force-field parameters
Next, one-step perturbation was used to investigate the effect of changing individual parameters on the NOE distance bound violations of the $\beta$-peptide. The following solute parameters were separately varied: backbone partial charges, force constants of backbone torsional-angle energy terms (changes up to $\pm 20 \%$ ), and $\mathrm{C} 12$ repulsive van der Waals parameters of GROMOS atom types (Oostenbrink et al. 2004) C, O, N, CH1, CH2, and $\mathrm{CH} 3$ (changes up to $\pm 10 \%$ ). The results for changes from the 53A6 force field are shown in Fig. 3. The biggest effect comes from the change in backbone atomic partial charges. Increasing them improves the agreement with the experimental NOE
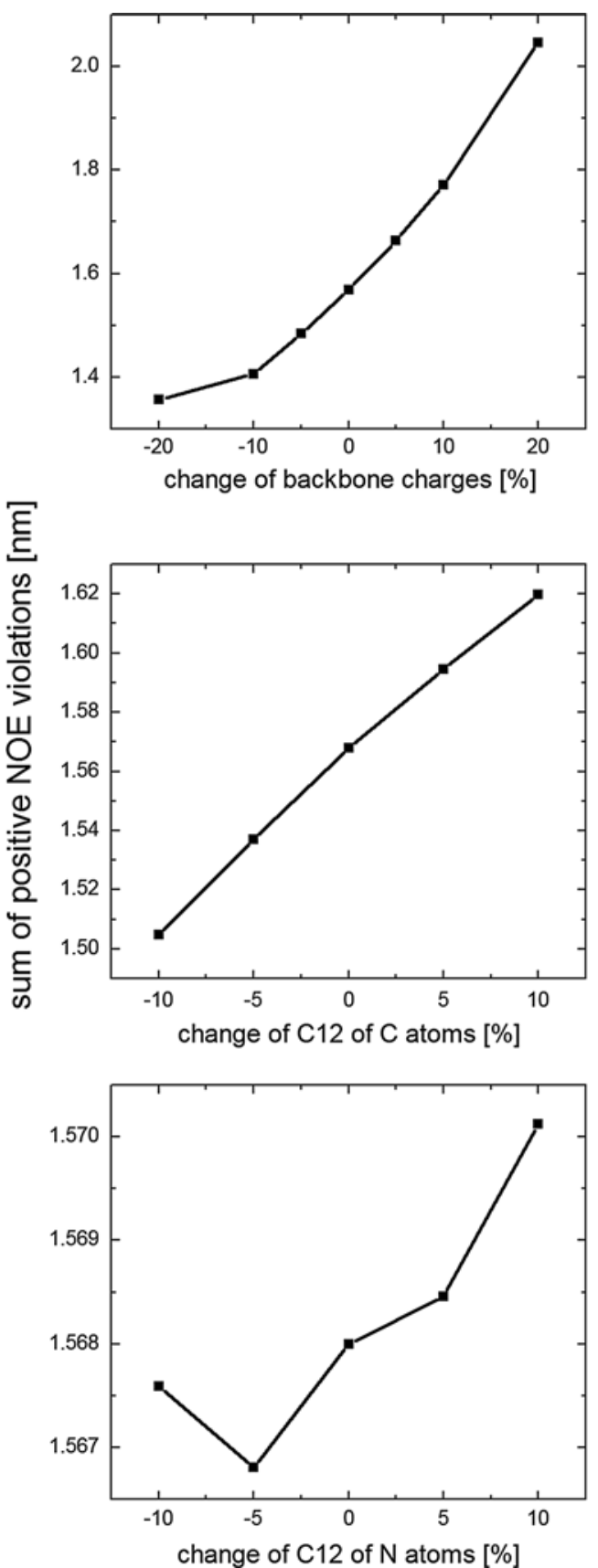
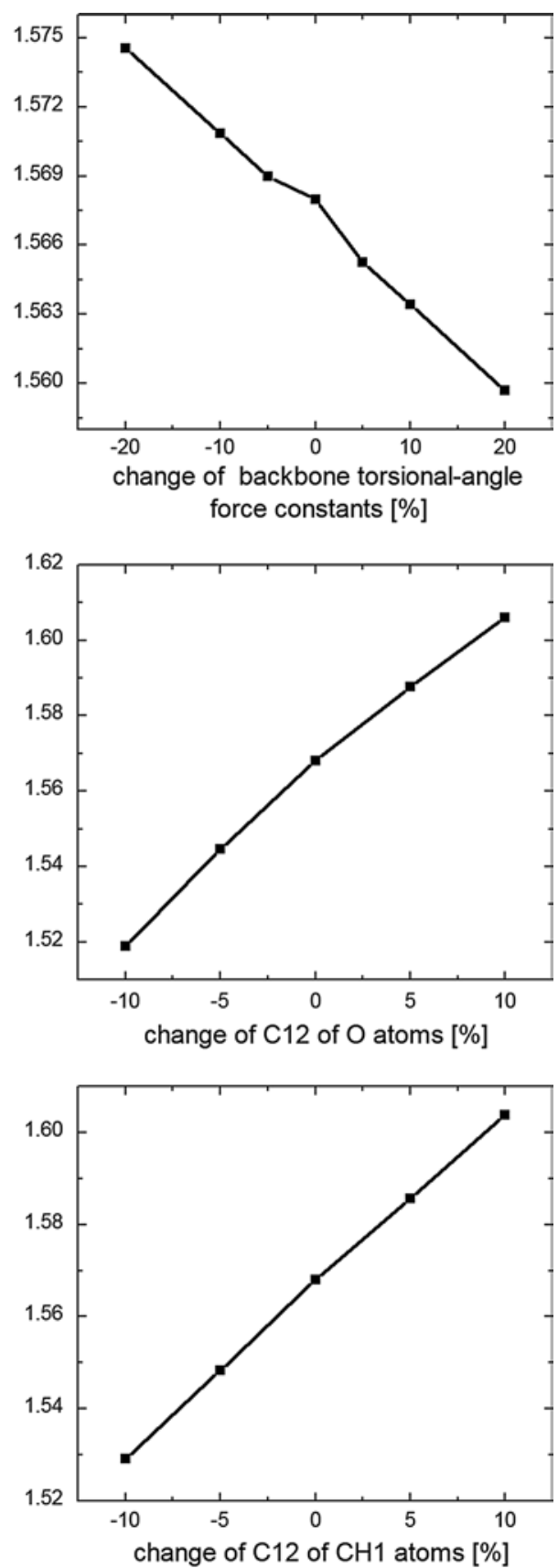
data, and vice versa. This may be due to stabilization of the helical conformation of the $\beta$-peptide over the nonhelical ones by the increase of backbone partial charges. Increasing or decreasing the force constants of backbone torsionalangle energy terms has only a very small effect on improving or impairing the agreement with the experimental data. Decreasing the repulsive $\mathrm{C} 12$ van der Waals parameters improves the agreement except for the atom type $\mathrm{CH} 3$.

For the hepta- $\alpha$-peptide, similar conclusions were obtained for the changes in backbone torsional-angle force constants and $\mathrm{C} 12$ parameters of the backbone atoms (Fig. 4). However, an opposite relation between force-field parameters and NOE violations was observed for the change of backbone partial charges; i.e., reducing the backbone partial charges improves the agreement with the experimental NOE data for the $\alpha$-peptide. This may be caused by the $\alpha$-peptide adopting predominantly unfolded conformations (Wang et al. 2010). Increasing the backbone partial charges destabilizes unfolded conformations over helical ones, and thus impairs the agreement with experimental data for the $\alpha$-peptide while it improves the agreement for the $\beta$-peptide. This observation nicely demonstrates that force-field parameters should not be obtained based on single simulations of macromolecules. A quantity $Q\left(\vec{r}^{N}\right)$ may pose conflicting requirements on force-field parameters for different systems, and different quantities $Q\left(\vec{r}^{N}\right)$ may show conflicting requirements for a single system. This illustrates the challenge of transferable force-field development.

In summary, one-step perturbation, which in this case is equivalent to reweighting configurations, constitutes an efficient technique to predict violations of NOE distance bounds of peptides upon changing force-field parameters. However, a necessary condition is that conformations relevant to both Hamiltonians be present in the conformational ensemble used to obtain $\langle Q\rangle$ values. Reweighting can also be used to predict quantities $\langle Q\rangle$ other than NOE distance bound violations such as X-ray diffraction intensities, residential dipolar couplings, or circular dichroism (CD) spectra for a particular system, which makes it a powerful technique for force-field development that easily reduces the number of required separate simulations by an order of magnitude.

Acknowledgments This work was financially supported by the National Center of Competence in Research (NCCR) in Structural Biology and by grant number 200020-137827 of the Swiss National Science Foundation, and by grant number 228076 of the European Research Council (ERC), which is gratefully acknowledged.

\section{References}

Bachmann S, Dolenc J, van Gunsteren WF (2013) On the use of onestep perturbation to investigate the dependence of different properties of liquid water upon a variation of model parameters from a single simulation. Mol Phys 111(14-15):2334
Cornell WD, Cieplak P, Bayly CI, Gould IR, Merz KM, Ferguson DM, Spellmeyer DC, Fox T, Caldwell JW, Kollman PA (1995) A second generation force field for the simulation of proteins, nucleic acids, and organic molecules. J Am Chem Soc 117(19):5179-5197

Daura X, Gademann K, Jaun B, Seebach D, van Gunsteren WF, Mark AE (1999) Peptide folding: when simulation meets experiment. Angew Chem Int Ed 38(1-2):236-240

Eichenberger AP, Gattin Z, Yalak G, van Gunsteren WF (2010) Molecular dynamics simulation of ester-linked hen egg white lysozyme reveals the effect of missing backbone hydrogen bond donors on the protein structure. Helv Chim Acta 93(10):1857-1869

Eichenberger AP, Allison JR, Dolenc J, Geerke DP, Horta BAC, Meier K, Oostenbrink C, Schmid N, Steiner D, Wang DQ, van Gunsteren WF (2011) GROMOS plus plus software for the analysis of biomolecular simulation trajectories. J Chem Theory Comput 7(10):3379-3390

Horta BAC, Fuchs PFJ, van Gunsteren WF, Hünenberger PH (2011) New interaction parameters for oxygen compounds in the GROMOS force field: improved pure-liquid and solvation properties for alcohols, ethers, aldehydes, ketones, carboxylic acids, and esters. J Chem Theory Comput 7(4):1016-1031

Hritz J, Oostenbrink C (2009) Efficient free energy calculations for compounds with multiple stable conformations separated by high energy barriers. J Phys Chem B 113(38):12711-12720

Jorgensen WL, Maxwell DS, TiradoRives J (1996) Development and testing of the OPLS all-atom force field on conformational energetics and properties of organic liquids. J Am Chem Soc 118(45):11225-11236

Li DW, Brüschweiler R (2010) NMR-based protein potentials. Angew Chem Int Ed 49(38):6778-6780

Lin ZX, van Gunsteren WF (2013) Refinement of the application of the GROMOS 54A7 force field to $\beta$-peptides. J Comput Chem 34(32):2796-2805

Liu HY, Mark AE, van Gunsteren WF (1996) Estimating the relative free energy of different molecular states with respect to a single reference state. J Phys Chem 100(22):9485-9494

MacKerell AD, Bashford D, Bellott M, Dunbrack RL, Evanseck JD, Field MJ, Fischer S, Gao J, Guo H, Ha S, Joseph-McCarthy D, Kuchnir L, Kuczera K, Lau FTK, Mattos C, Michnick S, Ngo T, Nguyen DT, Prodhom B, Reiher WE, Roux B, Schlenkrich M, Smith JC, Stote R, Straub J, Watanabe M, Wiorkiewicz-Kuczera J, Yin D, Karplus M (1998) All-atom empirical potential for molecular modeling and dynamics studies of proteins. J Phys Chem B 102(18):3586-3616

Oostenbrink C (2012) Free energy calculations from one-step perturbations. In: Baron R (ed) Computational drug discovery and design, methods in molecular biology, vol 819. Humana, New York, pp 487-499

Oostenbrink C, van Gunsteren WF (2005) Efficient calculation of many stacking and pairing free energies in DNA from a few molecular dynamics simulations. Chem Eur J 11(15):4340-4348

Oostenbrink C, Villa A, Mark AE, van Gunsteren WF (2004) A biomolecular force field based on the free enthalpy of hydration and solvation: the GROMOS force-field parameter sets 53A5 and 53A6. J Comput Chem 25(13):1656-1676

Reif MM, Hünenberger PH, Oostenbrink C (2012) New interaction parameters for charged amino acid side chains in the GROMOS force field. J Chem Theory Comput 8(10):3705-3723

Reif MM, Winger M, Oostenbrink C (2013) Testing of the GROMOS force-field parameter set 54A8: structural properties of electrolyte solutions, lipid bilayers, and proteins. J Chem Theory Comput $9(2): 1247-1264$

Riniker S, Christ CD, Hansen HS, Hünenberger PH, Oostenbrink C, Steiner D, van Gunsteren WF (2011) Calculation of relative free energies for ligand-protein binding, solvation, and 
conformational transitions using the GROMOS software. J Phys Chem B 115(46):13570-13577

Schmid N, Allison JR, Dolenc J, Eichenberger AP, Kunz APE, van Gunsteren WF (2011a) Biomolecular structure refinement using the GROMOS simulation software. J Biomol NMR 51(3):265-281

Schmid N, Eichenberger AP, Choutko A, Riniker S, Winger M, Mark AE, van Gunsteren WF (2011b) Definition and testing of the GROMOS force-field versions 54A7 and 54B7. Eur Biophys J 40(7):843-856

Schmid N, Christ CD, Christen M, Eichenberger AP, van Gunsteren WF (2012) Architecture, implementation and parallelisation of the GROMOS software for biomolecular simulation. Comput Phys Commun 183(4):890-903

Schuler LD, Daura X, van Gunsteren WF (2001) An improved GROMOS96 force field for aliphatic hydrocarbons in the condensed phase. J Comput Chem 22(11):1205-1218

Seebach D, Abele S, Gademann K, Guichard G, Hintermann T, Jaun B, Matthews JL, Schreiber JV (1998) $\beta 2$ - and $\beta 3$-peptides with proteinaceous side chains: synthesis and solution structures of constitutional isomers, a novel helical secondary structure and the influence of solvation and hydrophobic interactions on folding. Helv Chim Acta 81(5):932-982

Seebach D, Mathad RI, Kimmerlin T, Mahajan YR, Bindschadler P, Rueping M, Jaun B, Hilty C, Etezady-Esfarjani T (2005) NMR-solution structures in methanol of an $\alpha$-heptapeptide, of a $\beta 3 / \beta 2$-nonapeptide, and of an all- $\beta 3$-icosapeptide carrying the 20 proteinogenic side chains. Helv Chim Acta 88(7):1969-1982

Steiner D, Allison JR, Eichenberger AP, van Gunsteren WF (2012) On the calculation of ${ }^{3} \mathrm{~J} \alpha \beta$-coupling constants for side chains in proteins. J Biomol NMR 53(3):223-246

Torrie GM, Valleau JP (1977) Nonphysical sampling distributions in Monte Carlo free-energy estimation: umbrella sampling. J Comput Phys 23(2):187-199

van Gunsteren WF, Berendsen HJC (1990) Computer-simulation of molecular-dynamics: methodology, applications, and perspectives in chemistry. Angew Chem Int Ed 29(9):992-1023

van Gunsteren WF, Bakowies D, Baron R, Chandrasekhar I, Christen M, Daura X, Gee P, Geerke DP, Glättli A, Hünenberger PH, Kastenholz MA, Oostenbrink C, Schenk M, Trzesniak D, van der Vegt NFA, Yu HB (2006) Biomolecular modeling: goals, problems, perspectives. Angew Chem Int Ed 45(25):4064-4092

van Gunsteren WF et al. http://www.gromos.net

Wang DQ, Friedmann M, Gattin Z, Jaun B, van Gunsteren WF (2010) The propensity of $\alpha$-aminoisobutyric acid (=2-methylalanine; Aib) to induce helical secondary structure in an $\alpha$-heptapeptide: a computational study. Helv Chim Acta 93(8):1513-1531

Wüthrich K, Billeter M, Braun W (1983) Pseudo-structures for the 20 common amino acids for use in studies of protein conformations by measurements of intramolecular proton-proton distance constraints with nuclear magnetic resonance. J Mol Biol 169(4):949-961 